\title{
EXPERIENCIAS DE PAZ EN CONFLICTOS FRONTERIZOS POR EL AGUA
}

\author{
Mario Hernán López Becerra \\ Profesor Universidad de Caldas Departamento de Economía y Administración, \\ mariohl@une.net.co
}

Manizales, 2009-05-06 (Rev. 2009-06-07)

\section{RESUMEN}

El artículo aborda algunos de los conflictos recientes surgidos entre países por la disputa de los recursos hídricos compartidos, en especial por los ríos fronterizos. A pesar de las complejas problemáticas entre países fronterizos que, en algunos casos, convocan múltiples formas de violencia como camino para tramitar las diferencias de intereses, en la realidad emergen diversas experiencias de paz que permiten generar y mantener acuerdos sobre el uso y el acceso al agua. Estas experiencias de paz pueden señalarse como formas de paz imperfecta.

En la primera parte del artículo se presenta un marco general de los conflictos recientes entre países como producto de diversos intereses frente al tema del agua; en la segunda parte se realiza una revisión de la problemática ambiental en el marco de los estudios actuales sobre la paz; por último, se presentan experiencias de paz entre los países que disputan recursos hídricos, reportadas por organismos de las Naciones Unidas y ordenadas con base en la matriz propuesta por el profesor Francisco Muñoz de la Universidad de Granada.

\section{PALABRAS CLAVE}

Paz imperfecta, recursos hídricos compartidos, conflictos, problemática ambiental.

\section{PEACE EXPERIENCES IN BORDER CONFLICTS DUE TO WATER}

\section{ABSTRACT}

This document describes some international conflicts that are a consequence of the fight for shared hydrological resources, especially rivers located at international borders. It is a paradox that, despite the complex problems between bordering countries that negotiate through violence in conflicts of interests, many peace experiences emerge in reality in order to generate and maintain agreements concerning the use and access to water. These peace experiences can be called "imperfect peace experiences". The first part of the text presents a general context of recent international conflicts as a result of different interests regarding water-related topics. Secondly, a review of the environmental problems within the framework of current studies concerning peace is carried out. Finally, some of the peace experiences between countries in dispute over hydrological resources are introduced, reported by different organizations belonging to the United Nations, and are then ordered according to the model proposed by Francisco Muñoz, professor of the Universidad de Granada.

\section{KEY WORDS}

Imperfect peace, shared hydrological resources, conflicts, environmental problems. 


\section{LOS CONFLICTOS ACTUALES POR LOS RECURSOS HÍDRICOS}

Como se ha señalado profusamente, el hallazgo más inquietante del siglo $X X$ tiene que ver con el descubrimiento de la fragilidad del planeta tierra, una paradoja que opaca el esfuerzo de la civilización occidental por construir el presente y el futuro con base en una única versión del desarrollo. Las transformaciones ambientales a nivel local y global se pueden constatar en la presencia de fenómenos como el incremento de la temperatura del planeta, la disminución de la capa de ozono y los procesos de desertificación, deforestación y agotamiento de las fuentes de agua.

Si bien las civilizaciones occidentales se han construido en torno al agua -bajo ideologías que han considerado a la naturaleza como fuente ilimitada de recursos-, los procesos de deforestación, contaminación y los cambios en los usos del agua han generado una creciente escasez que, en algunos puntos del planeta, es notablemente crítica. La sociedad occidental, soportada en una ideología urbano-industrial, la ha convertido en un bien estratégico escaso y comercializable, fundamental para garantizar la sostenibilidad de los programas de desarrollo. Los usos insostenibles del agua (contaminación, pobreza, presión excesiva sobre las fuentes, desertificación, deforestación, entre otros) la han transformado en un "recurso escaso", susceptible de generar múltiples conflictos entre países que hacen parte de una misma cuenca, comunidades aledañas o actores económicos y sociales que se abastecen de una fuente hídrica común.

Pedro Arrojo Agudo, de la Universidad de Zaragoza (2009), presenta indicadores de la crisis actual en materia de disponibilidad y acceso de las poblaciones al suministro del agua y sus efectos sobre la calidad de la vida. De acuerdo con Arrojo, más de 1.100 millones de personas no tienen acceso a una red de abastecimiento de agua potable adecuada, a las que habría que sumar otros 2.400 millones de personas que no tienen acceso a un saneamiento adecuado. En el siglo XX la población mundial se triplicó, dando lugar a un aumento del $600 \%$ en el consumo de agua y desencadenando las primeras crisis y conflictos a causa del agua. Durante el siglo XXI la población aumentará en un 40$50 \%$ a la vez que, regiones que actualmente tienen un bajo desarrollo, liderarán el crecimiento y la industrialización ${ }^{1}$.

Para el Grupo de Procesos y Sistemas de Ingeniería Ambiental de la Universidad Autónoma de Madrid (2007), durante el siglo XXI la escasez de agua conducirá a un recrudecimiento de los conflictos por los recursos hídricos compartidos. El mismo grupo de investigación advierte que la combinación del aumento de población, las expectativas de mejora de la calidad de vida, los planes regionales de desarrollo (basados en la disponibilidad del agua) y el reparto desigual de los recursos acuáticos, dará lugar a una demanda y una escasez de agua sin precedentes, con graves consecuencias ambientales, sociales, económicas y políticas. La Organización de las Naciones Unidas predice que el acceso al agua será la mayor causa de conflictos y guerras en África durante los próximos 25 años, especialmente en regiones en las que varios países comparten ríos y lagos. Para señalar los alcances de la crisis del agua en el planeta, el mismo grupo de investigadores advierte que "la degradación sistemática y generalizada de los ecosistemas acuáticos continentales tiene como una de sus consecuencias que 1.200 millones de personas no tengan acceso a aguas potables. De mantenerse las tendencias en curso, serán más de 4.000 millones para $2025^{\prime 2}$.

De acuerdo con las Naciones Unidas (2006), existen 263 cuencas internacionales que atraviesan fronteras políticas de dos o más países:

"Esas cuencas, en las que vive aproximadamente el $40 \%$ de la población del mundo, cubren casi la mitad de la superficie de la tierra y a ellas corresponde cerca del $60 \%$ de las corrientes de agua dulce del mundo. Un total de 145 naciones tienen parte de su territorio en cuencas internacionales y 21 países están situados enteramente en el interior de cuencas internacionales"3.

En el plano político, es posible rastrear conflictos activos entre países asociados con las disputas por el acceso compartido a las fuentes de agua; en la literatura consultada se encuentra información acerca de los conflictos entre Israel y Líbano por las aguas del río 
Jordán, de la India y Pakistán por las del Indo, de la India y Bangladesh por las del Ganges y de los Estados Unidos con México por el uso y acceso a las aguas del río Bravo. Como se verá más adelante, la mayoría de estos conflictos han encontrado soluciones por la vía diplomática y han garantizado el acceso de las poblaciones a las fuentes de agua, aún en medio de guerras, construyendo experiencias de paz imperfecta que deben ser examinadas y valoradas.

Por su parte, Diego Cevallos (2009) recoge algunos de los conflictos actuales entre países por el acceso y control de las fuentes de agua ${ }^{4}$. Según Cevallos, cincuenta y un países de todas las regiones del mundo están en riesgo de afrontar conflictos por el agua en los próximos 10 años. Algunos de los conflictos fronterizos por el acceso a las fuentes de agua son los siguientes ${ }^{5}$.

1. El aprovechamiento del las aguas del río Nilo para el desarrollo de regadíos y la generación de electricidad es una fuente de tensiones en la zona.

2. La pugna por las aguas del río Jordan sigue siendo un tema candente desde que en 1967 constituyó uno de los desencadenantes de la Guerra de los Seis Días.

3. El Líbano mantiene desde hace años la acusación de que Israel manipula el caudal del río Litani.

4. India mantiene una disputa con Pakistán por el control de las aguas del río Indo y con Bangladesh por las del Ganges.

5. Docenas de grandes ríos se encuentran secos antes de alcanzar el mar. Es el caso del río Nilo en Egipto, del río amarillo en China, del Indo en Pakistán, y de los ríos Grande y Colorado en Estados Unidos, entre otros.

Los ejemplos muestran los eventuales conflictos que se pueden generar al compartir los recursos hídricos entre dos o más países, esta situación se presenta en más de 263 cuencas hidrográficas de importancia mundial.

\section{RASGOS DE LA PROBLEMÀTICA AMBIENTAL EN CLAVE DE PAZ}

En su origen, los actuales conflictos ambientales están ligados a una visión reduccionista del desarrollo que ubica a la naturaleza en la condición de recurso económico, al mismo tiempo que impulsa una estrategia de crecimiento insostenible. Fenómenos complejos como el agotamiento de las fuentes de agua dulce, el incremento de la temperatura del planeta, la disminución de la capa de ozono y el deterioro generalizado de valiosos ecosistemas, han provocado una crisis ambiental de implicaciones globales. El examen de las causas del daño ambiental ha puesto en evidencia el carácter agónico de la racionalidad que soporta las ideologías del progreso. Como lo advierte Vicent Martínez ${ }^{6}$, no hay que temer al fracaso del desarrollo sino a su éxito.

La relación casi siempre conflictiva entre la naturaleza y las culturas obliga a dar un giro epistemológico hacia la sustentabilidad. Algunos de los ejes del giro epistemológico necesario para construir una nueva racionalidad, han sido propuestos por Martínez con claras alusiones a la problemática ambiental al advertir que la naturaleza debe dejar de ser distante y objetiva:

“(...) Frente a la objetividad: intersubjetividad e interpelación humana. Sustituir la perspectiva del observador distante por la de participante. El conocimiento deja de ser una relación entre sujeto y objeto para convertirse en una relación entre sujetos. No ser neutrales respecto de valores. Pasar al paradigma de la comunicación (...)"?

Los patrones actuales de producción, distribución y consumo sobrepasan los umbrales de irreversibilidad a partir de los cuales se generan los daños ambientales; esta tendencia implica el aumento en la complejidad del daño ambiental, multiplica la frecuencia y la 
magnitud de los impactos ambientales y acelera los cambios en las condiciones físicas del planeta, con efectos tales como la globalización de los conflictos asociados a la escasez del agua y la generación de luchas políticas por los "recursos estratégicos".

Una valoración general de la problemática ambiental del desarrollo lleva a concluir que las soluciones exclusivamente técnicas no han dado los resultados esperados; la ecología ha permitido constatar que los procesos biológicos necesitan un tiempo considerablemente mayor que el que les permite la presión humana; los sistemas sociales tienden a mantenerse a pesar de los indicadores; los conflictos ambientales son focos de luchas políticas $\mathrm{y}$, en consecuencia, es evidente que el progreso continuo ha aumentado los problemas ambientales generando una conflictividad que necesita ser resuelta apelando a diversas formas de cooperación pacífica ${ }^{8}$.

En consecuencia, una dimensión sustentable del desarrollo está asociada a la puesta en marcha de nuevas formas de producción y consumo, enmarcada en una racionalidad capaz de superar los dualismos entre la naturaleza y las culturas, lo cual implica generar transformaciones en la estructura social, política y económica y la movilización de la subjetividad, potenciando la capacidad altruista, transformadora, cooperativa, imaginativa, solidaria y pacifista de los seres humanos.

\section{CONFLICTO Y PAZ POR EL ACCESO A LAS FUENTES DE AGUA}

Según Aaron Wolf, director de la base de datos Conflictos Transfronterizos sobre el Agua en Estados Unidos, el único caso conocido de guerra por el agua se remonta a 4.500 años, cuando el uso de los ríos Tigris y Eufrates enfrentó a dos ciudades del actual Iraq. Pueblos rivales, como los de India y Pakistán o Israel y Palestina -dice Wolf- suelen resolver conflictos por el agua al mismo tiempo que luchan sin tregua en otros terrenos; se trata de una demostración clara de generación de alternativas pacifistas a la solución de conflictos y que coinciden con los estudios acerca de los procesos de paz imperfecta, en tanto "se trata de definir aquellos espacios e instancias en los que se pueden detectar acciones que crean paz, a pesar de que estén en contextos en los que existan conflictos y violencias"

La información aportada por el Departamento de Información Pública de las Naciones Unidas (2006), con relación a la forma pacífica de resolver o mitigar las controversias internacionales relacionadas con el agua, permite reconocer diversas experiencias de paz imperfecta, así como múltiples formas de mediación y acciones de empoderamiento pacifista. Se trata de casos en los cuales los actores políticos, institucionales y comunitarios optan por potenciar el desarrollo de las capacidades de los otros y alcanzan regulaciones pacíficas de los conflictos, a pesar de estar enmarcados, en casi todos los casos, en realidades profundamente afectadas por la violencia.

Para efectos de reconocer experiencias de resolución pacífica de conflictos asociados al tema del agua, a continuación se presentan diversos casos de conflictos internacionales derivados de la presencia de recursos hídricos compartidos. La información es tomada directamente de los documentos de Naciones Unidas ${ }^{10}$ y organizada en términos de las expresiones de paz imperfecta que se pueden reconocer, de las experiencias de mediación en los conflictos y de las diversas formas de empoderamiento pacifista por parte de las comunidades que tienen conflictos de esta naturaleza.

\section{LAS PACES IMPERFECTAS POR EL AGUA}

1. Entre los enemigos más acérrimos se han negociado acuerdos jurídicos para compartir el agua; estos acuerdo se han mantenido aunque persistan los conflictos con relación a otras cuestiones. Camboya, Laos, Tailandia y Vietnam, apoyados por las Naciones Unidas, han podido cooperar desde 1957 en el Marco de la Comisión del Río Mekong, anteriormente conocida como Comité del Mekong, y mantuvieron intercambios técnicos durante la guerra de Vietnam. 
2. Desde 1995, Israel y Jordania, con la participación de los Estados Unidos, han celebrado conversaciones periódicas para compartir las aguas del río Jordán, aun cuando estuvieran hasta época reciente en estado de guerra oficial. La Comisión del Río Indo, establecida con el apoyo del Banco Mundial, sobrevivió a dos guerras ente India y Pakistán.

\section{LOS TRATADOS INTERNACIONALES COMO INSTRUMENTOS DE MEDIACIÓN}

3. La historia de los tratados internacionales sobre el agua se remonta a 2500 A.C., cuando las dos ciudades-estados de Lagash y Umma, en Sumeria, establecieron un acuerdo para poner fin a una controversia sobre el agua a lo largo del río Tigris, que suele considerarse el primer tratado de la historia. Desde entonces, el conjunto de tratados sobre el agua que se han establecido es considerable.

4. Según la Organización de las Naciones Unidas para la Agricultura y la Alimentación, desde el año 85 de nuestra era se han redactado más de 3.600 tratados relacionados con recursos hídricos internacionales.

5. La Convención de las Naciones Unidas sobre el derecho de los usos de los cursos de agua internacionales para fines distintos de la navegación de 1997 es un instrumento internacional que se centra en la gestión de los recursos hídricos compartidos

\section{EL PAPEL DE LAS INSTITUCIONES EN LA MEDIACIÓN PARA LA PAZ}

6. En febrero de 1999 se acordó entre India y Pakistán establecer un marco para la cuenca del río Nilo, donde viven 160 millones de personas distribuidas en 10 países, a fin de luchar contra la pobreza y estimular el desarrollo económico en la región mediante la promoción de la utilización equitativa de los recursos hídricos comunes y la distribución equitativa de los beneficios. Esta iniciativa, apoyada por el Banco Mundial y el Programa de las Naciones Unidas para el Desarrollo, es un arreglo transitorio hasta que se establezca un marco permanente.

7. (Se requiere) una institución que fomente eficazmente un proceso de participación y compromiso a largo plazo, y el apoyo financiero adecuado de terceros que gozan de la confianza de todas las facciones. El proceso de examen y deliberación a menudo lleva tiempo -el acuerdo sobre el Indo llevó 10 años, en el caso del Ganges tomó 30 y el Jordán 40- debido a la necesidad de fomentar la confianza y una medida de participación en el proceso que los países participantes deberán sentir como propio.

\section{EL EMPODERAMIENTO PACIFISTA}

8. En los últimos 150 años sólo se han producido 37 controversias graves en que ha habido estallidos de violencia, en comparación con los 150 tratados que se han firmado. Las naciones valoran esos acuerdos porque contribuyen a aumentar la estabilidad y predictibilidad de las relaciones internacionales en lo atinente a los recursos hídricos.

9. En febrero de 1999 se acordó establecer un marco para la cuenca del río Nilo, donde viven 160 millones de personas distribuidas en 10 países, a fin de luchar contra la pobreza y estimular el desarrollo económico en la región mediante la promoción de la utilización equitativa de los recursos hídricos comunes y la distribución equitativa de los beneficios

\section{A MANERA DE CONCLUSIÓN}

La relación entre las culturas y la naturaleza es altamente conflictiva en razón a la ideología imperante del desarrollo que asume a la naturaleza como una gran despensa de bienes y servicios destinados a la satisfacción de las necesidades humanas. La actual crisis ambiental -al mismo tiempo global y local- interpela de manera profunda los términos actuales del desarrollo y su soporte en una racionalidad que agoniza. 
Es probable que la crisis ambiental del desarrollo obligue a redefinir la conflictividad como una noción exclusivamente antropocéntrica, para darle paso a una conflictividad biocéntrica de manera que el conflicto no se revele solamente a través de "las tensiones, divergencias de criterios, incluso de emociones y sentimientos entre los seres humanos". Una noción ampliada de la conflictividad involucraría todas las formas de vida y el conjunto del patrimonio natural, a partir de ahí se puede definir la cultura de paz como un cultivo de las relaciones con los otros y la naturaleza. Para el efecto -siguiendo a Francisco Muñoz ${ }^{11}$ - se requiere adoptar un punto de vista naturalista y complejo, ecológico; una teoría abierta de los conflictos, entendiéndolos en el marco de las dialécticas abiertas.

Los desafíos para avanzar hacia el desarrollo sostenible son los mismos que enfrenta el reconocimiento de las múltiples formas de paz que emergen en medio de los conflictos a la manera de paces imperfectas: en el terreno epistemológico ambas perspectivas requieren la gestión de la complejidad, la comprensión de las emergencias positivas y negativas, la superación de la fragmentación del conocimiento -rompiendo el falso dualismo cultura/naturaleza- y el reconocimiento de la transdisciplinariedad, la plurimetodología y la transculturalidad en los distintos abordajes que se realicen para la comprensión de la realidad y la realización de acciones que favorezcan la gestión pacífica y el empoderamiento pacifista.

La interpretación crítica de la problemática ambiental del desarrollo se nutre de la complejidad, de los sistemas abiertos, de los equilibrios dinámicos y busca superar la fragmentación del conocimiento como también lo hace la paz imperfecta. El estudio de los conflictos por el agua permite identificar esas coincidencias, con lo cual se abre una ruta de análisis que eventualmente puede convocar al estudio de una paz ambiental imperfecta capaz de reconocer y dinamizar las experiencias de sustentabilidad ambiental que realizan múltiples actores en diversos contextos, esto implicaría trabajar en la definición de paz ambiental como correlato del conflicto ambiental. Quedan por examinar los componentes axiológicos de esa aproximación.

En el caso de los conflictos fronterizos entre países que comparten recursos hídricos (particularmente los que tienen ríos por fronteras), vale la pena destacar las culturas de paz que se derivan de la multiplicidad de soluciones pacíficas: desde el año 85 de nuestra era se han redactado más de 3.600 tratados relacionados con recursos hídricos internacionales. Los países, aún en momentos o estados violencia, mantienen sus acuerdos para el acceso y el uso de las fuentes hídricas, en una demostración clara de la efectividad de las mediaciones y del empoderamiento pacifista. Como se señaló anteriormente, se trata de casos en los cuales diversos actores políticos, institucionales y comunitarios optan por potenciar el desarrollo de las capacidades de los otros y alcanzan regulaciones pacíficas de los conflictos, a pesar de estar enmarcados, casi todos los casos, en realidades profundamente afectadas por la violencia.

\section{NOTAS}

1. Arrojo, Pedro. Conflictos por el agua en el mundo. En Red: http://www.ecodes.org/pages/especial/alianzaporelagua/index.asp

2. Grupo de Procesos y Sistemas de Ingeniería Ambiental. El siglo XXI será el de los conflictos por el agua. Universidad de Zaragoza. 2007. En red. http://weblogs.madrimasd.org/remtavares/archive/2008/06/26/95546.aspx

\section{En: http://www.un.org/spanish/waterforlifedecade/}

4. Este marco de conflictos por el agua está ampliamente señalado en un artículo de Diego Cevallos sobre el tema de los conflictos internacionales actuales por el agua 
publicado en Tierramérica. Señala Cevallos que la falta de un elemento tan básico como el agua podría causar guerras internacionales. (...) "El agua podría ser el móvil de las guerras del siglo XXI, señalaron en numerosas ocasiones expertos y funcionarios de la Organización de Naciones Unidas. Aunque no todos comparten esa afirmación, pocos dudan de que el agua es una fuente de crecientes conflictos".

5. Ibíd.

6. Martínez, Vicent (2000). Saber hacer las paces: Epistemología de los estudios para la paz. Convergencia. Universidad Autónoma del Estado de México.

7. Ibid

8. López, Mario Hernán- Villegas, Guillermo (2006). La gestión ambiental de las Pymes en Manizales. El Interés ambiental y el consumo socialmente responsable del empresario. Universidad de Caldas.

9. Molina, Beatriz. Muñoz, Francisco Ed. (2004). Manual de Paz y Conflictos. Instituto de la paz y los Conflictos. Universidad de Granada. Eirene.

10. ONU- Agua. Decenio Internacional para la acción. El agua fuente de vida 2005-2015. Agua $\sin$ fronteras. En red, http://www.un.org/spanish/waterforlifedecade/waterborders.html

11. Muñoz, Francisco (2004). Una paz compleja, conflictiva e imperfecta. Una paz compleja y conflictiva. Instituto de la paz y los conflictos. Universidad de Granada (en prensa). 